\title{
Health-related quality of life and distress in elderly vs. younger patients with high-grade glioma-results of a multicenter study
}

\author{
Mirjam Renovanz ${ }^{1,2} \cdot$ Anne-Katrin Hickmann ${ }^{3} \cdot$ Minou Nadji-Ohl ${ }^{4}$. Naureen Keric ${ }^{1}$ - Elke Weimann ${ }^{5}$. \\ Christian Rainer Wirtz ${ }^{6} \cdot$ Susanne Singer ${ }^{7} \cdot$ Florian Ringel $^{1} \cdot$ Jan Coburger $^{6}$
}

Received: 17 October 2019 / Accepted: 6 February 2020 / Published online: 14 February 2020

(C) The Author(s) 2020

\begin{abstract}
Objective Half of all newly diagnosed patients with glioblastoma are $>65$ years still with a poor prognosis. Preserving quality of life is of high importance. However, patient reported outcome (PRO) data in this patient group is rare. The aim was to compare health-related quality of life (HRQoL) and distress between elderly and younger patients with high-grade glioma (HGG).

Methods We used baseline data of a prospective study where HGG patients were enrolled from 4 hospitals. Distress was measured using the distress thermometer (DT), HRQoL using the European Organization for Research and Treatment of Cancer (EORTC) Quality of Life Core Questionnaire (QLQ-C30) plus brain module (BN20). We compared distress and HRQoL by age ( $\geq 65$ vs. $<65$ years), gender, performance score, and time since diagnosis using multivariate linear and logistic regressions.

Results A total of $n=93$ (30\%) out of $n=309$ patients were $\geq 65$ years (mean 70 years, range 65-86 years). Mean DT score of elderly patients (5.2, SD 2.6) was comparable with younger patients (4.9, SD 2.6). Elderly patients reported significantly lower global health (GHS, mean elderly vs. younger; 50.8 vs. $60.5, p=0.003$ ), worse physical (56.8 vs. $73.3, p<0.001)$ and lower cognitive functioning ( 51.1 vs. $63.2, p=0.002)$, worse fatigue ( $52.5 \mathrm{vs} .43 .5, p=0.042)$, and worse motor dysfunction ( 34.9 vs. 23.6, $p=0.030$ ). KPS and not age was consistently associated with HRQoL.

Conclusion Physical functioning was significantly reduced in the elderly compared with younger HGG patients, and at the same time, emotional functioning and DT scores were comparable. KPS shows a greater association with HRQoL than with calendric age in HGG patients reflecting the particular importance for adequate assessment of HRQoL and general condition in elderly patients.
\end{abstract}

Keywords Elderly patients $\cdot$ High-grade glioma $\cdot$ Glioma $\cdot$ Glioblastoma $\cdot$ Quality of life $\cdot$ Age $\cdot$ Karnofsky performance status · Distress

\section{Introduction}

High-grade gliomas (HGG) represent the majority of gliomas with glioblastomas showing an incidence of 3.19 (3.16-3.21) cases per 100,000 person years [1]. Half of all newly

Mirjam Renovanz

mirjam.renovanz@med.uni-tuebingen.de

1 Department of Neurosurgery, University Medical Center, Mainz, Germany

2 Division of Neuro-Oncology, University Medical Center Tubingen, Tübingen, Germany

3 Department of Neurosurgery, Kantonsspital St. Gallen, St. Gallen, Switzerland diagnosed patients with glioblastoma (GBM) are older than 65 years [2]. Although recently improved and cost-effective treatment schemes for elderly patients with GBM have been published, these patients are facing a particularly dismal prognosis with a median survival of less than 6 months [3-6]

4 Department of Neurosurgery, Klinikum Stuttgart, Stuttgart, Germany

5 Department of Neurology, RKH Kliniken Ludwigsburg, Ludwigsburg, Germany

6 Department of Neurosurgery, University Medical Center Ulm, Ulm, Germany

7 Division of Epidemiology and Health Services Research, Institute of Medical Biostatistics, Epidemiology and Informatics, University Medical Center, Mainz, Germany 
compared with younger healthier patients with more favorable outcomes and molecular marker profiles according to recent studies [2]. In many clinical trials, higher age is an exclusion criterion, and historically, many elderly patients received no tumor-specific therapy but best supportive care [7, 8]. Due to general condition (frailty) and comorbidities, treating elderly patients with HGG is challenging, and multidisciplinary approaches are required [9-11].

Therefore, preserving quality of life in elderly patients is of high importance particularly considering the short life expectancy. Distress and supportive care needs in HGG patients are high. They should be addressed early in the disease trajectory by psycho-oncologists but also supportive and palliative care services [12]. Additionally, quality of life during survival is equally important as the length of survival in the elderly population $[12,13]$. Furthermore, it has been reported that lower performance status, higher age, female gender, and shorter time since diagnosis can be associated with distress and reduced quality of life [5]. However, health-related quality of life (HRQoL) data is rare for elderly people with HGG, and as soon as they experience clinical decline due to disease progression, assessment becomes difficult $[2,5]$.

Application of patient-reported outcomes (PROs) has become essential in assessing patients' HRQoL, distress, and psychosocial burden, as well as supportive care needs [14]. Recently, it has been shown that monitoring symptoms via PRO measures can be very helpful for cancer patients and even influence survival [15-17]. As the elderly population in HGG in most of the studies is defined by an age $\geq 65$ years, HRQoL and distress in this population might be different than in younger patients below than 65 years. Furthermore, so far, no data focusing especially on elderly patients' HRQoL are available. Thus, a comparison might help clinicians in clinical daily practice to advise patients regarding this important issue.

Therefore, the aims of our study were to describe quality of life and distress in elderly patients with HGG and to compare the results with those of the younger ones focusing in psychological and physical issues. Furthermore, we aimed to investigate factors associated with HRQoL of HGG patients.

\section{Patients and method}

\section{Patients}

From March 2014 to October 2014 as well as April 2015 to June 2016, we conducted two prospective studies assessing HRQoL, distress, and supportive care needs in glioma patients. Patients at four German neuro-oncological centers were approached during their outpatient visits and asked to participate in the study as previously described [18-20]. Inclusion criteria data analysis was a diagnosis of glioma WHO grades III-IV regardless of disease stage (initial diagnosis or recurrent disease), absence of aphasia impairing communication or consent to the study, and given informed consent. Patients were asked to complete several PRO measures. Furthermore, demographic and clinical data were recorded in a database. If patients were assessed several times during the study, we only evaluated the first assessment per patient for this crosssectional analysis. Figure 1 shows the course of the study. When patients declined the participation, gender, age, diagnosis, and if possible reason were documented.

\section{Instruments}

\section{Distress thermometer}

The distress thermometer (DT) is a self-reporting screening instrument developed by the National Comprehensive Cancer Network to evaluate psychological distress on a visual analog scale ( $0-10$ points). A problem list with 40 items is included for patients to indicate the area of concern (family, financial, and physical). Studies have proven its acceptance in oncological patients, and Goebel and colleagues evaluated the German version for brain tumor patients. A score $\geq 6$ indicates a significant burden in brain tumor patients according to Goebel et al. [21].

\section{European Organization for Research and Treatment of Cancer quality of life core questionnaire accompanied by the brain-specific module}

The EORTC QLQ-C30 is a widely accepted questionnaire evaluating cancer patients' quality of life. Functions' symptom and the global health status (GHS) are investigated (physical (physf), role (rolef), emotional (emof), social (socf), and cognitive functioning (cogf), fatigue, nausea and vomiting, pain, dyspnea, insomnia, appetite loss, constipation, diarrhea, and financial difficulties). Its validity and reliability have been proven in numerous clinical studies, and it is available in 103 languages. The additional module for brain tumor patients (BN20) consists of 20 questions specifically assessing their symptoms (3 neurological deficit scales, 1 future uncertainty scale, treatment, and disease-related symptoms) [22, 23]. The scores were calculated according to the user manual. Each scale ranges from 0 to 100 , with higher scores indicating better functioning for functional scales and worse symptoms for symptom scales.

\section{Statistical analysis}

We performed a descriptive explorative assessment comparing scores of DT and subscales of EORTC instruments between the elderly and the younger group. The following parameters were considered to be potentially associated with 


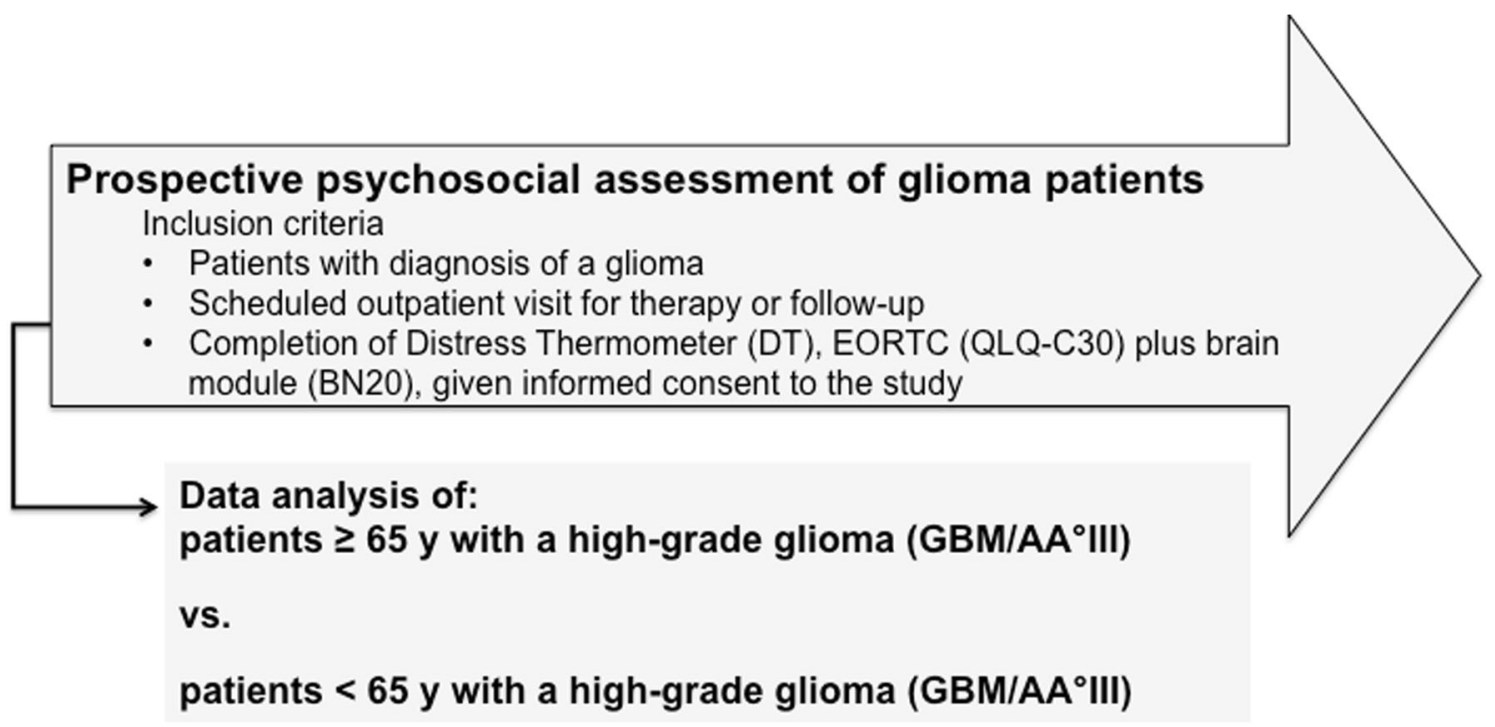

Outcomes:

- Scores of DT

- Subscales of EORTC instruments

- Multivariate analysis regarding clinical parameters: age $\geq 65 y$, KPS $\geq 70$, gender and time since diagnosis to assessment

Fig. 1 The course of the study

distress and quality of life: performance status (KPS) $\geq 70$, age ( $\geq 65$ years, according to clinical studies), gender, and time since diagnosis $[5,24]$. These variables were selected content driven by clinical relevance. We tested their association with univariate and multivariate models using binary logistic regression for distress (DT $\geq 6$ ) and linear regression for quality of life (all EORTC subscales).

\section{Ethics}

The study was in accordance with national law, institutional ethical standards, and the 1964 Helsinki Declaration and its later amendments. The ethic committees of all study centers approved the study (Mainz, Ludwigsburg, Stuttgart, and Ulm/ Gunzburg, Germany, No: 837.349 .15 (10117), and 837.472.13 (9157-F)). Patients provided written informed consent prior to data acquisition.

\section{Results}

\section{Patients}

A total of $n=93(30 \%)$ out of $n=309$ patients were $\geq 65$ years (mean $=70$ years, range $65-86$ years). The majority of the elderly population harbored a glioblastoma (GBM, $n=77$, $83 \%), n=53(57 \%)$ were male, median KPS was 70 (range 40-100), and mean time since diagnosis was 2.2 months. The younger population had a mean age of 48 years (range 19-64), $n=126$ were male (57\%), less patients had a GBM $(n=103$, $47 \%$ ), and the median KPS was 80 (range 40-100). Details are provided in Table 1.

\section{Distress and health-related quality of life}

Mean DT scores of elderly patients were comparable with those of younger patients (mean elderly patients vs. younger patients; $5.2(\mathrm{SD}=2.6)$ vs. 4.9 ( $\mathrm{SD}=2.6), p=0.42)$.

Elderly patients reported significantly lower GHS (mean elderly vs. younger; 50.8 vs. $60.5, p=0.003$ ), lower physf (56.8 vs. $73.3, p<0.001)$, lower $\operatorname{cogf}(51.1$ vs. $63.2, p=$ $0.002)$, higher fatigue ( 52.5 vs. $43.5, p=0.042)$, greater impairment by visual disorders ( $23.9 \mathrm{vs} .15 .0, p=0.013$ ), by motor dysfunction ( 34.9 vs. 23.6, $p=0.030$ ), by weakness of legs (31.4 vs. $20.8, p=0.03$ ), and greater problems regarding bladder control (22.3 vs. $10.8, p=0.001)$. Younger patients reported significantly higher symptom scores for insomnia (elderly patients vs. younger patients; 27.0 vs. $36.4, p=0.003$ ) and financial difficulties $(18.8$ vs. $33.8, p<0.001)$, please see Table 2. 
Table 1 Clinical and demographic data of the patient sample. All variables are indicated in numbers and \% in brackets if not otherwise specified

\begin{tabular}{|c|c|c|c|}
\hline Variable & All patients $n=309(100 \%)$ & Older patients $n=95(100 \%)$ & Younger patients $n=214(100 \%)$ \\
\hline \multicolumn{4}{|l|}{ Age in years } \\
\hline Mean (SD) & $55(14)$ & $70(4.5)$ & $48(10)$ \\
\hline Median (range) & $55(19-86)$ & $69(65-86)$ & $50(19-64)$ \\
\hline \multicolumn{4}{|l|}{ Gender } \\
\hline Male & $179(58)$ & $54(57)$ & $127(59)$ \\
\hline Female & $130(42)$ & $41(43)$ & $87(41)$ \\
\hline \multicolumn{4}{|l|}{ Living situation } \\
\hline Single & $66(21)$ & $16(17)$ & $48(23)$ \\
\hline In relationship & $229(74)$ & $76(80)$ & $155(72)$ \\
\hline Missing & $14(5)$ & $3(3)$ & $11(5)$ \\
\hline \multicolumn{4}{|l|}{ Working situation } \\
\hline Working & $170(55)$ & $11(12)$ & $140(65)$ \\
\hline Retired or early retired & $93(30)$ & $68(71)$ & $44(21)$ \\
\hline Missing & $46(15)$ & $16(17)$ & $30(14)$ \\
\hline \multicolumn{4}{|l|}{ WHO grade } \\
\hline WHO III & $128(41)$ & $17(18)$ & $110(51)$ \\
\hline WHO IV & $181(59)$ & $78(82)$ & $104(49)$ \\
\hline \multicolumn{4}{|l|}{ Survival at assessment } \\
\hline$<1$ year & $128(41)$ & $52(55)$ & $76(36)$ \\
\hline$>1$ and $<2$ years & $47(15)$ & $17(18)$ & $30(14)$ \\
\hline$>2$ years & $134(43)$ & $26(27)$ & $108(50)$ \\
\hline \multicolumn{4}{|l|}{ Tumor localization } \\
\hline Frontal & $128(41)$ & $37(39)$ & $91(43)$ \\
\hline Temporal & $88(28)$ & $28(29)$ & $60(28)$ \\
\hline Parietal & $21(7)$ & $7(7)$ & $14(6)$ \\
\hline Occipital & $48(16)$ & $16(18)$ & $32(15)$ \\
\hline Other unknown & $20(7) 4(1)$ & 7 (7) 0 & $13(6) 4(2)$ \\
\hline \multicolumn{4}{|c|}{ MGMT promotor methylation } \\
\hline Unmethylated & $128(41)$ & $46(48)$ & $82(38)$ \\
\hline Methylated & $86(28)$ & $32(34)$ & $54(26)$ \\
\hline Not analyzed & $73(24)$ & $14(15)$ & $59(28)$ \\
\hline Missing & $22(7)$ & $3(3)$ & $19(8)$ \\
\hline \multicolumn{4}{|l|}{ IDH status } \\
\hline IDH wild type & $127(41)$ & $56(59)$ & $71(33)$ \\
\hline IDH mutated & $84(27)$ & $20(21)$ & $64(30)$ \\
\hline Not analyzed & $76(25)$ & $16(17)$ & $60(28)$ \\
\hline Missing & $22(7)$ & $3(3)$ & $19(9)$ \\
\hline \multicolumn{4}{|l|}{ Ongoing chemotherapy } \\
\hline Yes & $125(40)$ & $53(56)$ & $72(34)$ \\
\hline No & $158(51)$ & $39(41)$ & $119(56)$ \\
\hline Missing & $26(9)$ & $3(3)$ & $23(10)$ \\
\hline \multicolumn{4}{|l|}{ Surgery for recurrent tumor } \\
\hline Yes & $87(28)$ & $14(15)$ & $73(34)$ \\
\hline No & $188(61)$ & $67(71)$ & $121(57)$ \\
\hline Missing & $34(11)$ & $14(15)$ & $20(8)$ \\
\hline \multicolumn{4}{|l|}{ Karnofsky index } \\
\hline Mean (SD) & $78(16)$ & $74(18)$ & $81(15)$ \\
\hline Median (range) & $80(30-100)$ & $70(40-100)$ & $80(30-100)$ \\
\hline
\end{tabular}

Time since diagnosis in months 
Table 1 (continued)

\begin{tabular}{llll}
\hline Variable & All patients $n=309(100 \%)$ & Older patients $n=95(100 \%)$ & Younger patients $n=214(100 \%)$ \\
\hline Mean (SD) & $42(51)$ & $25(44)$ & $49(54)$ \\
Median (range) & $19(0.5-298)$ & $10(0.5-288)$ & $31(0.5-296)$ \\
\hline
\end{tabular}

\section{Factors associated with distress and quality of life}

In general, KPS was consistently associated with HRQoL regarding all functioning scales (physical functioning, $p<0.0001,95 \%$ CI 30.73, 47.35; emotional functioning, $p<0.0001,95 \%$ CI 9.29, 25.59; cognitive functioning, $p<0.0001,95 \%$ CI 13.18, 33.72; social functioning, $p<0.0001,95 \%$ CI $24.14,45.48)$. This was true for most of the symptom scales as well as single item scales. Age same or above 65 years was associated with worse physical functioning $(p=0.029$, $95 \%$ CI $-15.66,-0.84)$, less financial difficulties $(p<0.0001,95 \%$ CI $-29.99,-9.36)$, visual disorders $(p=0.028,95 \%$ CI $0.77,13.48)$, and seizures ( $p=$ $0.030,95 \%$ CI $-14.52,-0.75)$. Details are provided in Table 3.

\section{Discussion}

In our analysis comparing elderly and younger HGG patients, we found that the elderly population seems to be more affected by the disease than younger ones with regard to physical impairment. We found significant differences in scores between the two patient groups possibly highlighting the frailty of many elderly patients. However, regarding emotional function and DT, the results were comparable in both groups.

\section{Study population and generalizability of the data}

Although this is one of the first studies describing HRQoL in HGG with a focus on age, we have to take into account that according to a former drop-out analysis of the study, $30 \%$ of all patients seen within the screening period declined the
Table 2 Comparison between patients $\geq 65$ and $<65$ years regarding score on DT and subscale or symptom scale on EORTC instruments

\begin{tabular}{|c|c|c|c|}
\hline $\begin{array}{l}\text { Score on distress thermometer resp. } \\
\text { subscale or symptom scale on EORTC instruments }\end{array}$ & $\geq 65$ years mean $(\mathrm{SD})$ & $\begin{array}{l}<65 \text { years mean } \\
(\mathrm{SD})\end{array}$ & $p$ \\
\hline Distress score & $5.2(2.6)$ & $4.9(2.6)$ & 0.42 \\
\hline Global health status (GHS) & $50.8(22.1)$ & $60.5(22.6)$ & 0.003 \\
\hline Physical functioning & $56.8(33.6)$ & $73.3(27.1)$ & $<0.0001$ \\
\hline Role functioning & $52.6(38.8)$ & $60.1(34.4)$ & 0.21 \\
\hline Emotional functioning & $62.3(26.6)$ & $60.0(26.5)$ & 0.66 \\
\hline Social functioning & $52.5(35.4)$ & $57.6(34.8)$ & 0.35 \\
\hline Cognitive functioning & $51.1(33.8)$ & $63.2(31.4)$ & 0.002 \\
\hline Fatigue & $52.5(30.6)$ & $43.5(29.3)$ & 0.042 \\
\hline Nausea & $7.7(17.4)$ & $8.3(16.5)$ & 0.49 \\
\hline Pain & $24.9(30.8)$ & $24.2(29.8)$ & 0.702 \\
\hline Insomnia & $27.0(32.1)$ & $36.4(35.2)$ & 0.003 \\
\hline Appetite loss & $18.7(28.8)$ & $19.2(30.3)$ & 0.701 \\
\hline Constipation & $18.2(29.9)$ & $15.5(27.6)$ & 0.69 \\
\hline Financial difficulties & $18.8(29.1)$ & $33.8(37.5)$ & $<0.0001$ \\
\hline Future uncertainty & $45.3(30.4)$ & $42.5(29.2)$ & 0.58 \\
\hline Visual disorder & $23.9(26.1)$ & $15.0(20.8)$ & 0.013 \\
\hline Motor dysfunction & $34.9(31.5)$ & $23.6(25.7)$ & 0.030 \\
\hline Communication deficit & $35.7(33.8)$ & $28.4(29.8)$ & 0.40 \\
\hline Headaches & $24.6(33.3)$ & $30.4(33.9)$ & 0.06 \\
\hline Seizures & $5.4(34.6)$ & $8.5(23.0)$ & 0.20 \\
\hline Drowsiness & 48.9 (34.6) & $42.9(31.6)$ & 0.57 \\
\hline Itchy Skin & $23.1(30.1)$ & $16.3(26.2)$ & 0.25 \\
\hline Hair loss & $19.7(32.2)$ & $16.2(31.1)$ & 0.38 \\
\hline Weakness of legs & $31.4(36.1)$ & $20.8(31.3)$ & 0.03 \\
\hline Bladder control & $22.3(33.8)$ & $10.8(25.3)$ & 0.001 \\
\hline
\end{tabular}


Table 3 Results of the multivariate analysis regarding the EORTC subscales and single items with content-driven-selected variables

\begin{tabular}{|c|c|c|c|c|c|}
\hline Subscale or symptom & Variable & Beta & $p$ & $95 \%$ CI lower & 95\% CI upper \\
\hline \multirow[t]{4}{*}{ Physf } & Age $\geq 65($ yes/no $)$ & -0.126 & 0.029 & -15.66 & -0.84 \\
\hline & Time since diagnosis & 0.040 & 0.484 & -0.53 & 1.12 \\
\hline & KPS $\geq 70($ yes/no $)$ & 0.526 & $<0.0001$ & 30.73 & 47.35 \\
\hline & gender & -0.060 & 0.278 & -10.58 & 3.05 \\
\hline \multirow[t]{4}{*}{ Rolef } & Age $\geq 65$ (yes/no) & 0.015 & 0.815 & -15.66 & -0.84 \\
\hline & Time since diagnosis & 0.113 & 0.072 & -.53 & 1.12 \\
\hline & KPS $\geq 70($ yes/no $)$ & 0.416 & $<0.0001$ & 30.73 & 47.35 \\
\hline & Gender & -0.033 & 0.592 & -10.58 & 3.05 \\
\hline \multirow[t]{4}{*}{ Emof } & Age $\geq 65$ (yes/no) & 0.102 & 0.130 & -1.65 & 12.82 \\
\hline & Time since diagnosis & 0.030 & 0.650 & -0.63 & 1.00 \\
\hline & KPS $\geq 70($ yes/no $)$ & 0.278 & $<0.0001$ & 9.29 & 25.59 \\
\hline & Gender & -0.175 & 0.007 & -15.86 & -2.51 \\
\hline \multirow[t]{4}{*}{$\operatorname{Cogf}$} & Age $\geq 65$ (yes/no) & -0.084 & 0.205 & -15.01 & 3.24 \\
\hline & Time since diagnosis & 0.071 & 0.280 & -0.47 & 1.60 \\
\hline & KPS $\geq 70($ yes/no $)$ & 0.294 & $<0.0001$ & 13.18 & 33.72 \\
\hline & Gender & -0.080 & 0.213 & -13.75 & 3.08 \\
\hline \multirow[t]{4}{*}{ Socf } & Age $\geq 65$ (yes/no) & 0.011 & 0.863 & -8.60 & 10.24 \\
\hline & Time since diagnosis & 0.083 & 0.191 & -0.37 & 1.77 \\
\hline & KPS $\geq 70($ yes/no $)$ & 0.408 & $<0.0001$ & 24.14 & 45.48 \\
\hline & Gender & -0.065 & 0.294 & -13.32 & 4.05 \\
\hline \multirow[t]{4}{*}{ Fatigue } & Age $\geq 65$ (yes/no) & 0.057 & 0.391 & -4.70 & 11.97 \\
\hline & Time since diagnosis & -0.094 & 0.147 & -1.61 & 0.24 \\
\hline & KPS $\geq 70($ yes/no $)$ & -0.291 & $<0.0001$ & -30.51 & -11.83 \\
\hline & Gender & 0.120 & 0.061 & -0.34 & 14.99 \\
\hline \multirow[t]{4}{*}{ Nausea } & Age $\geq 65$ (yes/no) & -0.060 & 0.388 & -7.19 & 2.81 \\
\hline & Time since diagnosis & 0.014 & 0.840 & -0.50 & 0.61 \\
\hline & KPS $\geq 70$ (yes/no) & -0.021 & 0.757 & -6.49 & 4.73 \\
\hline & Gender & 0.156 & 0.021 & 0.84 & 10.01 \\
\hline \multirow[t]{4}{*}{ Pain } & Age $\geq 65$ (yes/no) & 0.028 & 0.690 & -6.93 & 10.45 \\
\hline & Time since diagnosis & 0.082 & 0.226 & -0.37 & 1.56 \\
\hline & KPS $\geq 70$ (yes/no) & -0.133 & 0.053 & -19.38 & 0.120 \\
\hline & Gender & 0.098 & 0.143 & -2.03 & 13.92 \\
\hline \multirow[t]{4}{*}{ Dyspnea } & Age $\geq 65$ (yes/no) & 0.087 & 0.212 & -3.16 & 14.15 \\
\hline & Time since diagnosis & -0.044 & 0.519 & -1.28 & 0.65 \\
\hline & KPS $\geq 70($ yes/no $)$ & -0.134 & 0.050 & -19.39 & 0.02 \\
\hline & Gender & 0.029 & 0.669 & -6.23 & 9.69 \\
\hline \multirow[t]{4}{*}{ Insomnia } & Age $\geq 65($ yes/no $)$ & -0.125 & 0.074 & -18.96 & 0.88 \\
\hline & Time since diagnosis & -0.019 & 0.785 & -1.25 & 0.95 \\
\hline & KPS $\geq 70($ yes/no $)$ & -0.077 & 0.264 & -17.44 & 4.80 \\
\hline & Gender & 0.123 & 0.069 & -0.65 & 17.59 \\
\hline \multirow[t]{4}{*}{ Appetite loss } & Age $\geq 65$ (yes/no) & -0.114 & 0.093 & -16.87 & 1.31 \\
\hline & Time since diagnosis & -0.130 & 0.053 & -2.01 & 0.01 \\
\hline & KPS $\geq 70($ yes/no $)$ & -0.210 & 0.002 & -26.47 & -6.08 \\
\hline & Gender & 0.114 & 0.082 & -0.95 & 15.73 \\
\hline \multirow[t]{4}{*}{ Constipation } & Age $\geq 65$ (yes/no) & 0.022 & 0.754 & -7.29 & 10.04 \\
\hline & Time since diagnosis & -0.006 & 0.935 & -1.02 & 0.94 \\
\hline & KPS $\geq 70($ yes/no $)$ & -0.092 & 0.185 & -16.35 & 3.17 \\
\hline & Gender & 0.106 & 0.117 & -1.61 & 14.35 \\
\hline Diarrhea & Age $\geq 65($ yes/no $)$ & -0.074 & 0.296 & -9.41 & 2.88 \\
\hline
\end{tabular}


Table 3 (continued)

\begin{tabular}{|c|c|c|c|c|c|}
\hline Subscale or symptom & Variable & Beta & $p$ & $95 \%$ CI lower & 95\% CI upper \\
\hline & Time since diagnosis & -0.058 & 0.399 & -0.99 & 0.40 \\
\hline & KPS $\geq 70($ yes/no $)$ & -0.065 & 0.349 & -10.29 & 3.65 \\
\hline & Gender & 0.011 & 0.868 & -5.17 & 6.13 \\
\hline \multirow[t]{4}{*}{ Financial difficulties } & Age $\geq 65($ yes/no $)$ & -0.257 & $<0.0001$ & -29.95 & -9.36 \\
\hline & Time since diagnosis & -0.055 & 0.416 & -1.64 & 0.68 \\
\hline & KPS $\geq 70($ yes/no $)$ & -0.140 & 0.038 & -23.91 & -0.66 \\
\hline & Gender & -0.040 & 0.542 & -12.42 & 6.55 \\
\hline \multirow[t]{4}{*}{ GHS } & Age $\geq 65$ (yes/no) & -0.125 & 0.058 & -12.28 & 0.202 \\
\hline & Time since diagnosis & 0.003 & 0.960 & -0.69 & 0.72 \\
\hline & KPS $\geq 70($ yes/no $)$ & 0.327 & $<0.0001$ & 11.07 & 25.19 \\
\hline & Gender & -0.022 & 0.726 & -6.78 & 4.73 \\
\hline \multirow[t]{4}{*}{ Future uncertainty } & Age $\geq 65$ (yes/no) & -0.072 & 0.287 & -12.95 & 3.85 \\
\hline & Time since diagnosis & -0.162 & 0.015 & -2.07 & -0.22 \\
\hline & KPS $\geq 70($ yes/no $)$ & -0.238 & $<0.0001$ & -26.59 & -7.68 \\
\hline & Gender & 0.018 & 0.780 & -6.60 & 8.78 \\
\hline \multirow[t]{4}{*}{ Visual disorder } & Age $\geq 65$ (yes/no) & 0.149 & 0.028 & 0.77 & 13.48 \\
\hline & Time since diagnosis & -0.048 & 0.465 & -0.96 & 0.44 \\
\hline & KPS $\geq 70($ yes/no $)$ & -0.221 & 0.001 & -19.30 & -4.94 \\
\hline & Gender & 0.058 & 0.373 & -3.18 & 8.45 \\
\hline \multirow[t]{4}{*}{ Motor dysfunction } & Age $\geq 65$ (yes/no) & 0.059 & 0.335 & -3.74 & 10.95 \\
\hline & Time since diagnosis & 0.004 & 0.943 & -0.78 & 0.84 \\
\hline & KPS $\geq 70($ yes/no $)$ & -0.493 & $<0.0001$ & -42.60 & -26.13 \\
\hline & Gender & 0.046 & 0.435 & -4.07 & 9.41 \\
\hline \multirow[t]{4}{*}{ Communication deficit } & Age $\geq 65$ (yes/no) & -0.001 & 0.990 & -8.88 & 8.76 \\
\hline & Time since diagnosis & 0.012 & 0.858 & -0.89 & 1.06 \\
\hline & KPS $\geq 70($ yes/no $)$ & -0.356 & $<0.0001$ & -37.21 & -17.45 \\
\hline & Gender & 0.000 & 0.999 & -8.08 & 8.07 \\
\hline \multirow[t]{4}{*}{ Headache } & Age $\geq 65$ (yes/no) & -0.082 & 0.238 & -15.76 & 3.94 \\
\hline & Time since diagnosis & 0.045 & 0.508 & -0.72 & 1.45 \\
\hline & KPS $\geq 70$ (yes/no) & -0.021 & 0.760 & -12.77 & 9.33 \\
\hline & Gender & 0.163 & 0.015 & 2.17 & 20.22 \\
\hline \multirow[t]{4}{*}{ Seizures } & Age $\geq 65$ (yes/no) & -0.151 & 0.030 & -14.52 & -0.75 \\
\hline & Time since diagnosis & 0.021 & 0.755 & -0.63 & 0.87 \\
\hline & $\mathrm{KPS} \geq 70(\mathrm{yes} / \mathrm{no})$ & -0.205 & 0.003 & -19.36 & -4.01 \\
\hline & Gender & -0.040 & 0.545 & -8.22 & 4.36 \\
\hline \multirow[t]{4}{*}{ Drowsiness } & Age $\geq 65$ (yes/no) & 0.060 & 0.378 & -5.12 & 13.42 \\
\hline & Time since diagnosis & 0.020 & 0.761 & -0.87 & 1.18 \\
\hline & KPS $\geq 70$ (yes/no) & -0.255 & $<0.0001$ & -30.39 & -9.60 \\
\hline & Gender & 0.012 & 0.850 & -7.68 & 9.32 \\
\hline \multirow[t]{4}{*}{ Itchy skin } & Age $\geq 65$ (yes/no) & 0.093 & 0.185 & -2.75 & 14.11 \\
\hline & Time since diagnosis & 0.023 & 0.740 & -0.78 & 1.10 \\
\hline & KPS $\geq 70($ yes/no $)$ & -0.111 & 0.109 & -17.17 & 1.73 \\
\hline & Gender & 0.079 & 0.242 & -3.14 & 12.35 \\
\hline \multirow[t]{4}{*}{ Hair loss } & Age $\geq 65($ yes/no $)$ & 0.020 & 0.768 & -7.85 & 10.61 \\
\hline & Time since diagnosis & 0.070 & 0.290 & -0.47 & 1.56 \\
\hline & KPS $\geq 70($ yes/no $)$ & -0.042 & 0.535 & -13.69 & 7.13 \\
\hline & Gender & 0.291 & $<0.0001$ & 20.71 & 27.64 \\
\hline \multirow[t]{2}{*}{ Weakness of legs } & Age $\geq 65$ (yes/no) & 0.062 & 0.360 & -5.23 & 14.33 \\
\hline & Time since diagnosis & -0.053 & 0.426 & -1.51 & 0.64 \\
\hline
\end{tabular}


Table 3 (continued)

\begin{tabular}{|c|c|c|c|c|c|}
\hline Subscale or symptom & Variable & Beta & $p$ & $95 \%$ CI lower & 95\% CI upper \\
\hline \multirow{6}{*}{ Bladder control } & KPS $\geq 70($ yes/no $)$ & -0.307 & $<0.0001$ & -36.83 & -14.88 \\
\hline & Gender & 0.038 & 0.557 & -6.25 & 11.56 \\
\hline & Age $\geq 65($ yes/no $)$ & 0.129 & 0.062 & -0.43 & 17.32 \\
\hline & Time since diagnosis & 0.032 & 0.638 & -0.74 & 1.21 \\
\hline & $\mathrm{KPS} \geq 70$ (yes/no) & -0.210 & 0.002 & -25.75 & -5.74 \\
\hline & Gender & -0.013 & 0.841 & -8.95 & 7.30 \\
\hline
\end{tabular}

assessment [25]. Most of the decliners harbored a GBM and were in a significantly worse general condition; age was not found to be associated with study decline. Therefore, we probably present positively biased data for the elderly, but also for the younger population due to the selection bias of the study. Furthermore, we observed significant differences between the younger and the older patient group regarding WHO grade resulting in a higher percentage of patients in the younger patient group with a longer survival at the assessment than the older ones. However, it has been shown that DT and HRQoL are not influenced by WHO grade, and even in patients with meningioma, a significant burden can be observed [26-29]. Due to its greater incidence with higher age, GBM is diagnosed in older people more frequently than in the younger population [1]. Similarly, there were more patients under chemotherapy in the older patient group (56 vs. $34 \%$ in the younger patient group). This should be considered influencing the HRQoL.

\section{Results of younger and elderly patients with HGG}

Both patient groups reported relatively low social and emotional functioning compared with the normal population reflecting the significance of the disease and the relevance of the topic [30]. In comparison with other cancer diagnoses, where younger patients are more distressed than elderly patients in general, our findings further emphasize how much more burdening a brain tumor diagnosis is for all age groups (REFs). Further, we found a significant association between longer time since diagnosis and lower future uncertainty in the regression analysis underlining the initial shock of the diagnosis and a certain adaption to the situation and the symptoms over time.

Elderly patients' HRQoL was more affected than the younger one's regarding physical impairment and motor dysfunction. However, emotional functioning was comparable. This is interesting and probably due to accentuated neurocognitive deficits in older patients, which permit full recognition of the severity of the situation. On the other hand, older patients may be more satisfied with their life lived thus far and less anxious about life-time lost due to the disease. Furthermore, elderly people could hesitate to allow depressive thoughts and try to present themselves strong, compliant, and positive in order to preserve their strength but also the support of the doctor as long as advanced care planning is not early enough provided [31, 32].

Yet, elderly patients reported significantly lower GHS, physical and cognitive functioning, greater fatigue, and impairment due to visual disorders as well as greater motor dysfunction meaning that they definitely perceived critical impairment, even though some may not be disease related. Although HRQoL assessed by the EORTC instruments is a subjective estimation, it remains sometimes unclear what the item or function does mean for the individual patient. Elderly patients may recognize their problems well but seem emotionally not as burdened as we would probably assume. Assessing quality of life by structured questionnaires like the EORTC instruments is a standardized approach where an individual weighting of issues is not intended in order to facilitate interindividual comparisons. However, the assessment with individual consideration of the variety of topics influencing HRQoL (individualized approach) is complex, hard to implement in clinical routine or even in studies, and data are challenging to compare between patients [33-35]. However, regarding the brain tumor patient population, it may be worth to develop alternative approaches in order to assess the meaning of HRQoL for different individuals, e.g., by developing instruments more focusing on their situation or signaling questions implementable into clinical routine in order to facilitate, refine, and individualize the assessment.

In line with results of emotional functioning, the DT was comparable between the two groups. Finally, to admit a psychological burden may be more difficult than a physical impairment. Nonetheless, since some of the deficits older patients experience, such as loss in motor function, visual disturbances, weakness of legs, and loss of bladder control, may not be solely disease related and already persistent for a longer period of time, patients may have gotten used to them and find them less worrisome. Additionally, losing bodily functions may be more acceptable when growing old, in general. Greater fatigue in older patients may explain lower rates of insomnia compared with younger patients. On the other hand, cancer patients can develop symptoms of fatigue and present with sleeping disorder at the same time, which occur in brain 
tumor patients frequently and are suboptimal detected and treated by the neuro-oncologists [36].

Younger patients reported more often financial difficulties than elderly people, which is a significant problem in HGG patients. They often lose their higher executive functions and are not able to work anymore as they used to before the disease, leading to financial restrictions with significant consequences for their family and caregivers, who often have to play a new part in the relationship. Older patients, receiving pension or living off of retirement funds, do not need to worry as much anymore about providing for themselves and their family.

\section{Significance of age and KPS}

Our regression analysis revealed that after applying several models, KPS was associated stronger with HRQoL than calendric age. This is probably not surprising. However, given the fact that the newer studies led to different treatment schemes based on age, e.g., for GBM, it seems to be debatable to assign patients to therapy schemes considering solely their age in light of our findings: deciding on a more or less aggressive treatment should be based not only on KPS or calendric age alone, but also on patient perceived HRQoL. Undoubtedly, especially in elderly HGG patients, we should include geriatric assessment what seems to be helpful in geriatric cancer patients in general $[37,38]$.

\section{Limitations and strengths of the study}

Of note, we have to discuss several flaws of the study. First, we report a post hoc analysis, what gives a retrospective character to the prospectively collected data. Therefore, findings have to be interpreted carefully. Second, as already mentioned, due to the time consuming assessment, we observed a selection bias, as patients in a worse clinical condition in both groups independently of age declined participation in the study. Therefore, we report data of a well-functioning cohort which may influence mean scores, yet, since patients are assumed to have dropped out in both groups, the general findings regarding between group differences should be valid. Further, as mentioned above, the two patient populations exhibited differences regarding WHO grade, ongoing chemotherapy, and mean time since diagnosis. However, in a recent published review including glioblastoma patients, the authors found that chemotherapy and radiotherapy may not have harmful effects on HRQoL in fit elderly cancer patients [39]. A further limitation of the study is that we did not assess the comorbidities of the patients, which may have also influenced the results in the two different groups overestimating the influence of the HGG diagnosis. Furthermore, quality of life is influenced by multiple factors in this group, of which not all could be assessed as we analyzed content-driven variables to reduce multiple testing. Therefore, results and comparisons have to be interpreted carefully.
Yet, the strength of this study is that rare data are available for this patient group, and our study may on the one hand provide a basis for decision-making in daily clinical practice and on the other hand motivate to conduct further clinical studies assessing HRQoL in elderly HGG patients.

\section{Conclusion}

Physical functioning was significantly reduced in the elderly compared with younger HGG patients. Emotional functioning and DT scores were comparable. KPS shows a greater association with HRQoL than with calendric age in HGG patients reflecting the particular importance for adequate assessment of HRQoL and general condition in elderly patients.

Acknowledgments We acknowledge all patients and caregivers for their study participation. Further, we acknowledge all medical students of our study group Karoline Kohlmann, Mareile Janko, Anne-Katrin Reuter, Linda Stöckelmaier, Katrin Nickel, Dorothea Maurer, and Heike Lahr for their work as the manuscript contents of parts of their dissertation. Further, we thank Sonja Grüninger, Monika Deininger, and Christoph Richter for their tremendous support.

Funding information Open Access funding provided by Projekt DEAL. The Friedhelm Frees Stiftung, Mainz, Germany, funded this project.

\section{Compliance with ethical standards}

The study was in accordance with national law, institutional ethical standards, and the 1964 Helsinki Declaration and its later amendments. The ethic committees of all study centers approved the study (Mainz, Ludwigsburg, Stuttgart, and Ulm/Gunzburg, Germany, No: 837.349.15 (10117), and 837.472.13 (9157-F)). Patients provided written informed consent prior to data acquisition.

Conflict of interest The authors declare that they have no conflict of interest.

Open Access This article is licensed under a Creative Commons Attribution 4.0 International License, which permits use, sharing, adaptation, distribution and reproduction in any medium or format, as long as you give appropriate credit to the original author(s) and the source, provide a link to the Creative Commons licence, and indicate if changes were made. The images or other third party material in this article are included in the article's Creative Commons licence, unless indicated otherwise in a credit line to the material. If material is not included in the article's Creative Commons licence and your intended use is not permitted by statutory regulation or exceeds the permitted use, you will need to obtain permission directly from the copyright holder. To view a copy of this licence, visit http://creativecommons.org/licenses/by/4.0/.

\section{References}

1. Ostrom QT, Gittleman H, Truitt G, Boscia A, Kruchko C, Barnholtz-Sloan JS (2018) CBTRUS statistical report: primary brain and other central nervous system tumors diagnosed in the United States in 2011-2015. Neuro Oncol 20(suppl_4):iv1-iv86 
2. Ironside S, Das S, Sahgal A, Moroney C, Mainprize T, Perry JR (2017) Optimal therapies for newly diagnosed elderly patients with glioblastoma. Curr Treat Options in Oncol 18(11):66

3. Ghosh S, Baker S, de Castro DG, Kepka L, Kumar N, Sinaika V, Matiello J, Lomidze D, Dyttus-Cebulok K, Rosenblatt E, Fidarova E, Roa W (2018) Improved cost-effectiveness of short-course radiotherapy in elderly and/or frail patients with glioblastoma. Radiother Oncol 127(1):114-120

4. Moroney C, Perry JR, Tsang DS, Bilodeau D, Mueller C, Soliman H, Myrehaug S, Sahgal A, Tseng CL, Tsao MN (2017) Hospitalizations in elderly glioblastoma patients. Ann Palliat Med 6(Suppl 2):S161-S1S9

5. Perry JR, Laperriere N, O'Callaghan CJ, Brandes AA, Menten J, Phillips $C$ et al (2017) Short-course radiation plus temozolomide in elderly patients with glioblastoma. N Engl J Med 376(11):10271037

6. Wick W, Platten M, Meisner C, Felsberg J, Tabatabai G, Simon M, Nikkhah G, Papsdorf K, Steinbach JP, Sabel M, Combs SE, Vesper J, Braun C, Meixensberger J, Ketter R, Mayer-Steinacker R, Reifenberger G, Weller M, NOA-08 Study Group of Neurooncology Working Group (NOA) of German Cancer Society (2012) Temozolomide chemotherapy alone versus radiotherapy alone for malignant astrocytoma in the elderly: the NOA-08 randomised, phase 3 trial. Lancet Oncol 13(7):707-715

7. Amsbaugh MJ, Yusuf MB, Gaskins J, Burton EC, Woo SY (2017) Patterns of care and predictors of adjuvant therapies in elderly patients with glioblastoma: an analysis of the National Cancer Data Base. Cancer. 123(17):3277-3284

8. Iwamoto FM, Reiner AS, Panageas KS, Elkin EB, Abrey LE (2008) Patterns of care in elderly glioblastoma patients. Ann Neurol 64(6):628-634

9. Harris G, Jayamanne D, Wheeler H, Gzell C, Kastelan M, Schembri G et al (2017) Survival outcomes of elderly patients with glioblastoma multiforme in their 75 th year or older treated with adjuvant therapy. Int J Radiat Oncol Biol Phys 98(4):802-810

10. Tsang DS, Khan L, Perry JR, Soliman H, Sahgal A, Keith JL et al (2015) Survival outcomes in elderly patients with glioblastoma. Clin Oncol (R Coll Radiol) 27(3):176-183

11. Zouaoui S, Darlix A, Fabbro-Peray P, Mathieu-Daude H, Rigau V, Fabbro M et al (2014) Oncological patterns of care and outcomes for 265 elderly patients with newly diagnosed glioblastoma in France. Neurosurg Rev 37(3):415-423 discussion 23-4

12. Pace A, Villani V (2018) Palliative and supportive care of patients with intracranial glioma. Prog Neurol Surg 31:229-237

13. Harrison RA, de Groot JF (2018) Treatment of glioblastoma in the elderly. Drugs Aging 35(8):707-718

14. Dirven L, Aaronson NK, Heimans JJ, Taphoorn MJ (2014) Healthrelated quality of life in high-grade glioma patients. Chin J Cancer 33(1):40-45

15. Basch E, Deal AM, Dueck AC, Scher HI, Kris MG, Hudis C et al (2017) Overall survival results of a trial assessing patient-reported outcomes for symptom monitoring during routine cancer treatment. JAMA. 318(2):197-198

16. Basch E, Deal AM, Kris MG, Scher HI, Hudis CA, Sabbatini P et al (2016) Symptom monitoring with patient-reported outcomes during routine cancer treatment: a randomized controlled trial. J Clin Oncol 34(6):557-565

17. Satin JR, Linden W, Phillips MJ (2009) Depression as a predictor of disease progression and mortality in cancer patients: a meta-analysis. Cancer. 115(22):5349-5361

18. Hickmann AK, Hechtner M, Nadji-Ohl M, Janko M, Reuter AK, Kohlmann K, Haug M, Grüninger S, Deininger M, Ganslandt O, König J, Wirtz CR, Coburger J, Renovanz M (2017) Evaluating patients for psychosocial distress and supportive care needs based on health-related quality of life in primary brain tumors: a prospective multicenter analysis of patients with gliomas in an outpatient setting. J Neuro Oncol 131(1):135-151

19. Renovanz M, Hickmann AK, Coburger J, Kohlmann K, Janko M, Reuter AK et al (2018) Assessing psychological and supportive care needs in glioma patients - feasibility study on the use of the Supportive Care Needs Survey Short Form (SCNS-SF34-G) and the Supportive Care Needs Survey Screening Tool (SCNS-ST9) in clinical practice. Eur J Cancer Care 27(1)

20. Renovanz M, Maurer D, Lahr H, Weimann E, Deininger M, Wirtz CR et al (2018) Supportive care needs in glioma patients and their caregivers in clinical practice: results of a multicenter crosssectional study. Front Neurol 9:763

21. Goebel S, Mehdorn HM (2011) Measurement of psychological distress in patients with intracranial tumours: the NCCN distress thermometer. J Neuro Oncol 104(1):357-364

22. Aaronson NK, Ahmedzai S, Bergman B, Bullinger M, Cull A, Duez NJ, Filiberti A, Flechtner H, Fleishman SB, de Haes JC (1993) The European Organization for Research and Treatment of Cancer QLQ-C30: a quality-of-life instrument for use in international clinical trials in oncology. J Natl Cancer Inst 85(5):365-376

23. Taphoorn MJ, Claassens L, Aaronson NK, Coens C, Mauer M, Osoba D, Stupp R, Mirimanoff RO, van den Bent M, Bottomley A, EORTC Quality of Life Group, and Brain Cancer, NCIC and Radiotherapy Groups (2010) An international validation study of the EORTC brain cancer module (EORTC QLQ-BN20) for assessing health-related quality of life and symptoms in brain cancer patients. Eur J Cancer 46(6):1033-1040

24. Coomans M, Dirven L, K Aaronson N, Baumert BG, van den Bent M, Bottomley A et al (2019) The added value of health-related quality of life as a prognostic indicator of overall survival and progression-free survival in glioma patients: a meta-analysis based on individual patient data from randomised controlled trials. Eur J Cancer 116:190-198

25. Renovanz MHMK, Kohlmann K, Janko M, Nadji-Ohl M, Singer S, Ringel F, Coburger J, Hickmann AK (2017) Compliance with patient-reported outcome assessment in glioma patients: predictors for drop out. Neuro Oncol Practice

26. Goebel S, Mehdorn HM (2013) Development of anxiety and depression in patients with benign intracranial meningiomas: a prospective long-term study. Support Care Cancer 21(5):1365-1372

27. Boele FW, Zant M, Heine EC, Aaronson NK, Taphoorn MJ, Reijneveld JC, Postma TJ, Heimans JJ, Klein M (2014) The association between cognitive functioning and health-related quality of life in low-grade glioma patients. Neurooncol Pract 1(2):40-46

28. Fountain DM, Allen D, Joannides AJ, Nandi D, Santarius T, Chari A (2016) Reporting of patient-reported health-related quality of life in adults with diffuse low-grade glioma: a systematic review. Neuro Oncol 18(11):1475-1486

29. Zamanipoor Najafabadi AH, Peeters MCM, Dirven L, Lobatto DJ, Groen JL, Broekman MLD, Peerdeman SM, Peul WC, Taphoorn MJB, van Furth W (2017) Impaired health-related quality of life in meningioma patients-a systematic review. Neuro Oncol 19(7):897907

30. Schwarz R, Hinz A (2001) Reference data for the quality of life questionnaire EORTC QLQ-C30 in the general German population. Eur J Cancer 37(11):1345-1351

31. Koekkoek JA, Chang S, Taphoorn MJ (2016) Palliative care at the end-of-life in glioma patients. Handb Clin Neurol 134:315-326

32. Giammalva GR, Iacopino DG, Azzarello G, Gaggiotti C, Graziano F, Guli C et al (2018) End-of-life care in high-grade glioma patients. The palliative and supportive perspective. Brain Sci 8(7)

33. Hamidou Z, Baumstarck K, Chinot O, Barlesi F, Salas S, Leroy T, Auquier P (2017) Domains of quality of life freely expressed by 
cancer patients and their caregivers: contribution of the SEIQoL. Health Qual Life Outcomes 15(1):99

34. McGee HM, O'Boyle CA, Hickey A, O'Malley K, Joyce CR (1991) Assessing the quality of life of the individual: the SEIQoL with a healthy and a gastroenterology unit population. Psychol Med 21(3):749-759

35. Joyce CR, Hickey A, McGee HM, O'Boyle CA (2003) A theorybased method for the evaluation of individual quality of life: the SEIQoL. Qual Life Res Int J Qual Life Asp Treat Care Rehab 12(3): 275-280

36. Jeon MSD, Dhillon HM, Descallar J, Lam L, Allingham S, Koh ES, Currow DC, Agar MR (2019) Prevalence and severity of sleep difficulty in patients with a CNS cancer receiving palliative care in Australia. Neurooncol Pract:npz005
37. Goldberg RJ, Cullen LO (1986) Depression in geriatric cancer patients: guide to assessment and treatment. Hosp J 2(2):79-98

38. Kirkhus L, Saltyte Benth J, Gronberg BH, Hjermstad MJ, Rostoft S, Harneshaug M et al (2019) Frailty identified by geriatric assessment is associated with poor functioning, high symptom burden and increased risk of physical decline in older cancer patients: prospective observational study. Palliat Med 33(3):312-322

39. Cheng KK, Lim EY, Kanesvaran R (2018) Quality of life of elderly patients with solid tumours undergoing adjuvant cancer therapy: a systematic review. BMJ Open 8(1):e018101

Publisher's note Springer Nature remains neutral with regard to jurisdictional claims in published maps and institutional affiliations. 